\title{
Dental Care Expenditures and Retirement
}

\author{
Richard J. Manski, D.D.S., M.B.A., Ph.D. [Professor and Director], \\ Division of Health Services Research, Department of Health Promotion and Policy, Dental School, \\ University of Maryland \\ John Moeller, Ph.D. [Research Professor], \\ Division of Health Services Research, Department of Health Promotion and Policy, Dental School, \\ University of Maryland
}

Haiyan Chen, MD, Ph.D. [Assistant Research Professor],

Division of Health Services Research, Department of Health Promotion and Policy, Dental School, University of Maryland

Patricia A. St. Clair, Sc.B.,

RAND Corporation

Jody Schimmel, Ph.D.,

Mathematica Policy Research, Inc.

Larry Magder, MPH, Ph.D. [Associate Professor and Head], and

Division of Biostatistics, Epidemiology and Preventive Medicine, School of Medicine, University of Maryland

John V. Pepper, Ph.D.

Department of Economics, University of Virginia

\begin{abstract}
Objectives-To examine the relationship of dental care coverage, retirement, and out-of-pocket (OOP) dental expenditures in an aging population, using data from the Health and Retirement Study (HRS).

Methods-We estimate OOP dental expenditures among individuals who have dental utilization as a function of dental care coverage status, retirement, and individual and household characteristics. We also estimate a multivariate model controlling for potentially confounding variables.

Results-Overall, mean OOP dental expenditures among those with any spending were substantially larger for those without coverage than for those with coverage. However, controlling for coverage shows that there is little difference in spending by retirement status.

Conclusions-Although having dental coverage is a key determinant of the level of OOP expenditures on dental care; spending is higher among those without coverage than those who have dental insurance. We also found that while retirement has no independent effect on OOP dental expenditures once controlling for coverage, dental coverage rates are much lower among retirees.
\end{abstract}

\section{Keywords}

Dental; Utilization; Dentistry; Insurance; Coverage; Retirement

Mailing Address: Richard J. Manski, D.D.S., M.B.A., Ph.D., Professor and Director, Division of Health Services Research, Department of Health Promotion and Policy, Dental School, University of Maryland, 650 West Baltimore Street, Baltimore, Maryland 21201, 410-706-7245 (Voice) - 410-706-3028 (Fax), rmanski@umaryland.edu. 


\section{Introduction}

The transition from work to retirement affects household income and the time that individuals have available for non-work activities. In the United States, most people also experience a change in their health and dental insurance coverage around retirement. Prior to age 65 , the majority of individuals with coverage receive it through an employment setting, but beginning at age 65, virtually all Americans become universally covered by Medicare.

The coverage differences between employer-provided coverage and Medicare matter less for most types of health care than for dental care. Medicare provides generous coverage for many types of care, but it does not offer a comprehensive dental benefit [1], leaving many older Americans without any dental coverage. Further, many people retire prior to being eligible for Medicare [2], meaning that they potentially face several additional years without coverage. In the past, many relied on retiree health benefits to bridge the gap until Medicare, but this is becoming decreasingly likely as retiree health benefits continue to erode [3].

Previous work [4] has shown that only 38 percent of retired individuals have dental coverage, approximately $15 \%$ lower than those who are not retired, controlling for other individual and household factors known to affect the likelihood of coverage. Given this lower level of coverage, a concern is that retired individuals may not seek needed dental care [5]. This could occur because individuals do not seek any care at all, or because they forego more costly treatments that are recommended to them.

In terms of having any dental care use, retirees do not seem to have less use, despite their lower rates of coverage. In fact, Manski et al. [6] find that once controlling for other factors, individuals who are fully retired report rates of use that are $20 \%$ higher than those who are not retired at all. However, information about any use does not indicate how much care individuals are receiving or whether that care is relatively cheap or expensive.

This paper uses the 2006 Health and Retirement Study (HRS) to explore how retirement affects out-of-pocket dental care expenditures. The combination of decreased coverage and increased use, suggests that spending on dental care among those who have dental expenditures may increase after retirement. Given that this is a time when most households rely on a fixed income, large out-of-pocket spending on dental care could lead to important financial consequences for older adults.

\section{Methods}

The Health and Retirement Study (HRS) is a nationally representative longitudinal household survey that interviews individuals over age 50 and their spouses every two years; approximately 20,000 interviews are completed in each survey wave. Administered by the Institute for Social Research (ISR) at the University of Michigan and sponsored by the National Institute on Aging, the HRS is useful for the study of aging, retirement, and health among older populations in the United States.[2,7] ${ }^{1}$

Each HRS respondent is asked a large battery of questions including information about demographics; income and assets; physical and mental health; cognition, family structure and social supports; health care utilization and costs; health insurance coverage; labor force status and job history; and retirement planning and expectations. Because of the breadth of data available across health and labor force measures and the large sample of older Americans, the

\footnotetext{
${ }^{1}$ This analysis is based on data from the Early Release of the 2006 HRS. The Final Release of these data may contain slightly different data if errors in the Early Release data were detected, but we do not expect that these changes will substantively affect our results.
} 
HRS is the ideal data source for assessing the association between dental coverage, use, and retirement among an older population.

This analysis focuses on self reports in the HRS of the amount spent out-of-pocket for dental care during the two-year period prior to the most recent survey in 2006. As is common in analyses of health expenditures, we use the natural logarithm of expenditures, as this transformation places relatively little weight on higher expenditure values, which may be outliers and/or skew the results [8]. By using the natural log of expenditures, coefficient estimates can be interpreted as a percentage change in spending, as opposed to a dollar value change. All expenditure values are in 2006 dollars.

Rather than accounting for the probability of positive expenditures and the dollar value of expenditures among those with spending in a two-part or other model, we focus only on expenditures among those with OOP spending. In other words, our model conditions on having had some spending during the two-year period preceding 2006. We do this for several reasons. First, the HRS does not ask about out-of-pocket spending to individuals who report no use, meaning that we would be imputing data for those individuals. Second, the logarithm of zero is undefined, meaning that we would have had to transform our dependent variable in some way to account for cases with no spending. Third, and most importantly, in earlier work [6], we explored the effect of retirement on utilization, so we have already explored the first part of the two-part problem. Based on that work, we found that most people had some use, meaning that restricting only to people with OOP yields a sufficient sample size to focus specifically on expenditure levels.

HRS survey respondents are designated as fully retired if at the time of the survey interview they were not working for pay or self-employed and either (1) said that they were completely retired, or (2) reported their sole employment status as retired. Individuals are classified as partially retired if they were not fully retired but report retirement and either working or looking for work. Individuals not classified as fully or partly retired are designated as in the labor force if they report working for pay or report their labor force status as working full-time, part-time, or unemployed. Persons are classified as not retired and out of the labor force if they report being disabled and not in the labor force or never in the labor force.

Along with calculating the bivariate relationships between mean out-of-pocket dental expenditures, dental coverage, retirement status, and other person and household characteristics, we also estimate the natural logarithm regression model of the association outof-pocket dental care expenditures for various conditions of dental care coverage status with retirement status, controlling for other potentially confounding variables.

We used $\mathrm{z}$ tests to identify differences in mean OOP expenditures. Unless otherwise stated, all reported results are significant at the .05 level. The HRS core sample design is a multistage area probability sample of households, so all estimates and statistics reported were computed taking into account this design with the use of the software packages SUDAAN and STATA. $[9,10]$

\section{Results}

The 16,911 participants in the 2006 HRS represented 76,367,762 members of the communitybased population age 51 and above in that year, and comprise the study sample. Of these, more than half of the participants were female (58 percent, $\mathrm{N}=6,171)$. Ten percent $(\mathrm{N}=1,036)$ of the participants were non-Hispanic Black and 7 percent $(\mathrm{N}=698)$ were Hispanic. Twenty-five percent $(\mathrm{N}=2,635)$ of the participants were age 75 or older, 36 percent $(\mathrm{N}=3,764)$ were between the ages of 65 and 74 , and 40 percent $(\mathrm{N}=4,184)$ were between the ages of 51 and 64 . 
Approximately two-thirds of these participants reported some amount of out-of-pocket spending during the two year survey period ending in 2006. Among the sample with any expenditure, mean two-year spending was $\$ 951$. One half of the sample (50 percent, $N=5,287$ ) was fully retired (FR); 11 percent $(\mathrm{N}=1,133)$ partially retired $(\mathrm{PR}) ; 10$ percent $(\mathrm{N}=1,045)$ were not retired and in the labor force (NRILF); and 29 percent $(\mathrm{N}=3,118)$ were not retired and out of the labor force (NROLF).

\section{Descriptive Results}

Mean OOP dental expenditures among those with any spending were substantially larger for those without coverage than for those with coverage ( $\$ 1126$ vs. \$776, respectively). Stratifying the sample by coverage status takes into account the strong relationship between retirement and coverage which has been previously demonstrated [3], allowing isolation of the independent effect of retirement. Figure 1 presents mean OOP spending by coverage and retirement status. Once controlling for coverage, there is little difference in spending by retirement status.

Aside from coverage status, there are other characteristics that might affect spending. Table 1 shows the mean total OOP expenditure for those with dental expenditures, by dental coverage status and other characteristics. When not controlling for coverage status, there were no statistically meaningful differences in the mean level of out-of-pocket spending by retirement status, but there were other important differences by other characteristics, as shown in Table 1. Those who were older, White non-Hispanic, Hispanic, had higher income, more education, were widowed or divorced, or did not have teeth had higher out-of-pocket spending than their respective comparison categories. Differences in OOP dental expenditures by health status were not observed.

Mean out-of-pocket spending is higher among those without coverage in every instance in Table 1, showing the important reason for taking coverage into account. Stratification by dental coverage status also reveals differences by individual and household characteristics not observed in the full sample. Among those with coverage, White non-Hispanic and Hispanic older adults have higher OOP dental expenditures than Black non-Hispanics; this racial difference is not observed for those without coverage. Poor older adults without dental coverage have higher OOP expenditures for dental care than higher income older adults, but no such difference by income groups is observed for those with coverage. Similarly, no difference is observed among education categories for those with coverage, but high school graduate and college graduate adults have higher OOP expenditures for dental care than adults without a high school degree. Controlling for dental coverage, differences in OOP expenditures for dental care are not observed within age, sex, marital status, health status, or retirement status categories.

\section{Multivariate Results}

Table 2 presents the results of three regression models of the natural logarithm of out-of-pocket dental care expenditures, controlling for confounding factors that could influence the observed association between retirement status and dental expenditures. Similar to Table 1, the first model includes all participants, regardless of dental coverage status, though dental coverage status is included as a covariate. The second and third models are limited to participants with and without dental coverage respectively.

Controlling for confounders including coverage for the full sample, we do not find that retirement has any significant impact on OOP expenditures in the full model. Estimates obtained from the full model show that adults age sixty-five to seventy-four, adults without teeth, adults with a health status of fair or poor and adults without dental coverage are likely 
to have higher OOP expenditures for dental care than their respective comparison categories. On the other hand, Black non-Hispanic adults, poor adults, low and middle income adults, and those without a high school or college degree are likely to have lower OOP expenditures for dental care than their comparison groups.

In the model restricted to those with dental coverage, differences in education, the category poor income level, presence of teeth and the category health status fair or poor are no longer observed.

In the model restricted to those without dental coverage, differences are only observed for different levels of income, education and presence of teeth. Adults with lower income or less formal education are likely to have lower OOP expenditures for dental care than adults with higher income or more education. Also, in our model restricted to those without dental coverage adults without teeth are likely to have higher OOP expenditures for dental care than adults with teeth.

\section{Discussion}

This work shows that having dental coverage is a key determinant of the level of OOP expenditures on dental care; spending is $45 \%$ higher among those without coverage than those who have dental insurance. We also found that retirement has no independent effect on OOP dental expenditures once controlling for coverage. Given earlier work which showed that levels of any use were higher among retirees after controlling for coverage, why did we not observe increases in OOP dental spending? First, documented increases in use may have been offset by the use of reduced-cost or free clinics among retirees, who may have the additional time necessary to seek out such care. Second, it could be that retirees seek dental care initially, but forego more costly procedures if a dental provider recommends them. Overall, this could lead to similar levels of OOP expenditures but differing levels of any use by retirement status.

According to 2006 National Health Expenditures (NHE) data available from the Centers for Centers for Medicare \& Medicaid Services (CMS), approximately half of all dental expenditures are paid by private insurance [11]. While retirement does not have an independent effect on spending, dental coverage rates are much lower among retirees. Increasing coverage could be done by offering a dental benefit through Medicare, which the program currently lacks. However, our results show that even among retirees who have coverage and seek care, average OOP dental expenditures are nearly $\$ 400$ annually, which can be a large expense among those who live on a fixed income. Indeed, among the fully retired in our sample, the average household income was approximately $\$ 45,000$, meaning that OOP dental expenditures for a two-person household would consume almost $2 \%$ of annual income on average. OOP expenditures also vary by other individual and household characteristics, suggesting that targeting additional protections against high OOP dental spending to particular vulnerable populations could ease their burden. Reducing the cost of dental care may lead retirees to seek more appropriate care, resulting in better oral and overall health [12].

We caution that the expenditure levels reported in this study only apply to those individuals who seek dental care during a two-year period. Manski et al. [6] find that controlling for the same set of covariates as in this study, retirees are more likely to seek dental care. Thus, a higher fraction of retirees than non-retirees are likely to face the expenditures reported in this study, even though we did not find an independent effect of retirement on expenditure levels.

We were not able to discern in this study the types of services that individuals were using when spending out-of-pocket on dental care. New HRS data to be released in 2009 will contain more detailed information on dental utilization for a subset of respondents, and our future work will explore differences in utilization by retirement. We also intend to consider changes in dental 
coverage, use, and spending that occur around the transition from work to retirement. However, the data still will contain only self-reported data (less accurate than observational data) and will not contain information on the generosity of dental coverage which would allow us to better understand differences in OOP expenditures.

Despite some limitations, our results using the HRS appear to comport well with results of other studies. In earlier work [4,6], we showed that two-year use rates from the HRS fall within the bounds of estimates from the Medical Expenditure Panel Survey (MEPS), the National Health Interview Survey (NHIS), and the National Health and Nutrition Examination Survey (NHANES) [13]. For these reasons, we believe that information from the HRS about dental insurance coverage, service utilization, and OOP spending are the most reliable available for studying a national representation of the older population in the United States.

\section{Acknowledgments}

This investigation was supported by the National Institute on Aging of the National Institutes of Health. (R01 AG026090-01A2, Dental Coverage Transitions, Utilization and Retirement)

The HRS (Health and Retirement Study) is sponsored by the National Institute of Aging (grant number NIA U01AG009740) and is conducted by the University of Michigan.

\section{References}

1. Centers for Medicare \& Medicaid Services. Publication Number 10050. Baltimore, MD: Centers for Medicare \& Medicaid Services; 2008. Medicare \& You, 2009.

2. Growing Older in America: The Health and Retirement Study. Bethesda, MD: National Institute on Aging; 2007. Available at http://hrsonline.isr.umich.edu

3. The Kaiser Family Foundation and Health Research \& Educational Trust. Publication Number 7790. Menlo Park, CA: Kaiser Family Foundation; Sep 24. 2008 Employer Health Benefits: 2008 Annual Survey.

4. Manski RJ, Moeller JF, Chen H, St Clair PA, Schimmel J, Magder L, Pepper JV. Dental Care Coverage and Retirement. Journal of Public Health Dentistry. Submitted.

5. Manski RJ, Macek MD, Moeller JF. Private Dental Coverage: Who Has it and How Does it Influence Dental Visits and Expenditures? Journal of the American Dental Association November;2002 133(11): 1551-1559. [PubMed: 12462700]

6. Manski RJ, Moeller JF, Chen H, St Clair PA, Schimmel J, Magder L, Pepper JV. Dental Care Coverage and Utilization. Journal of Public Health Dentistry. Submitted.

7. RAND HRS Data, Version H. Produced by the RAND Center for the Study of Aging, with funding from the National Institute on Aging and the Social Security Administration; Santa Monica, CA: Feb. 2008

8. Wooldridge, Jeffrey. Introductory Econometrics. Mason, OH: Thomson-Southwestern; 2006. p. 197-200.

9. Research Triangle Institute. SUDAAN Software for analysis of correlated data. Release 6.40. Research Triangle Institute; 1995.

10. Statacorp. Stata Statistical Software: Release 7.0. College Station, TX: Stata Corporation; 2001.

11. Centers for Medicare \& Medicaid Services. Office of the Actuary, Table 8. National Health Expenditures (NHE); Jan. 2009

12. U.S. Department of Health and Human Services. Oral Health in America: A Report of the Surgeon General. Rockville, MD: U.S. Department of Health and Human Services, National Institute of Dental and Craniofacial Research, National Institutes of Health; 2000.

13. Macek MD, Manski RJ, Vargas CM, Moeller JF. Comparing Oral Health Care Utilization Estimates in the U.S. Across Three Nationally Representative Surveys. Health Services Research April;2002 37(2):499-521. [PubMed: 12036005] 


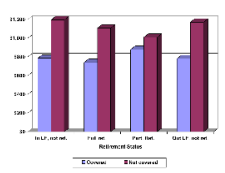

Figure 1. Weighted Estimates

Mean out-of-pocket dental expenditures in the two year period ending in 2006 for those with a dental visit, by coverage and retirement status 


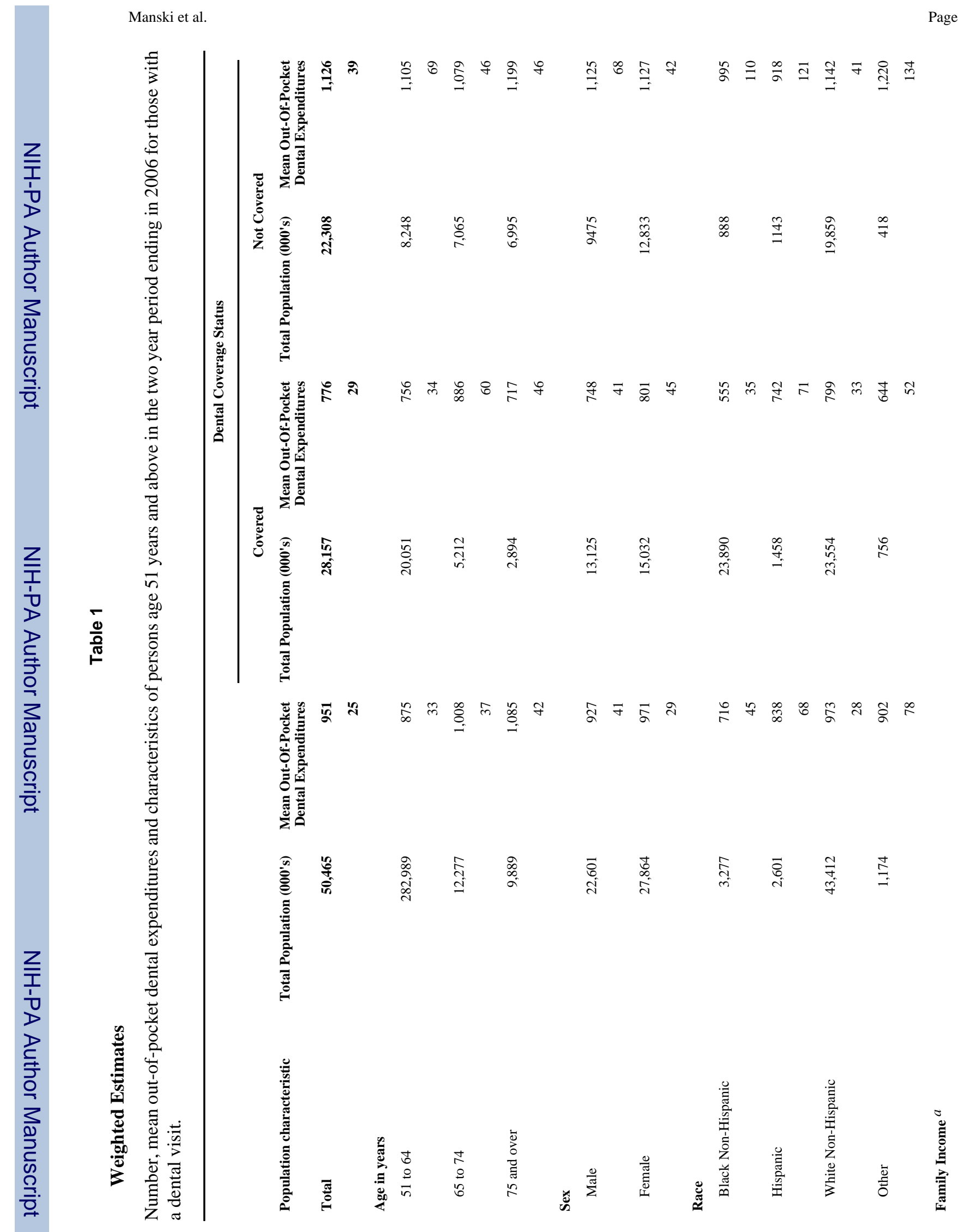

J Public Health Dent. Author manuscript; available in PMC 2010 October 19. 


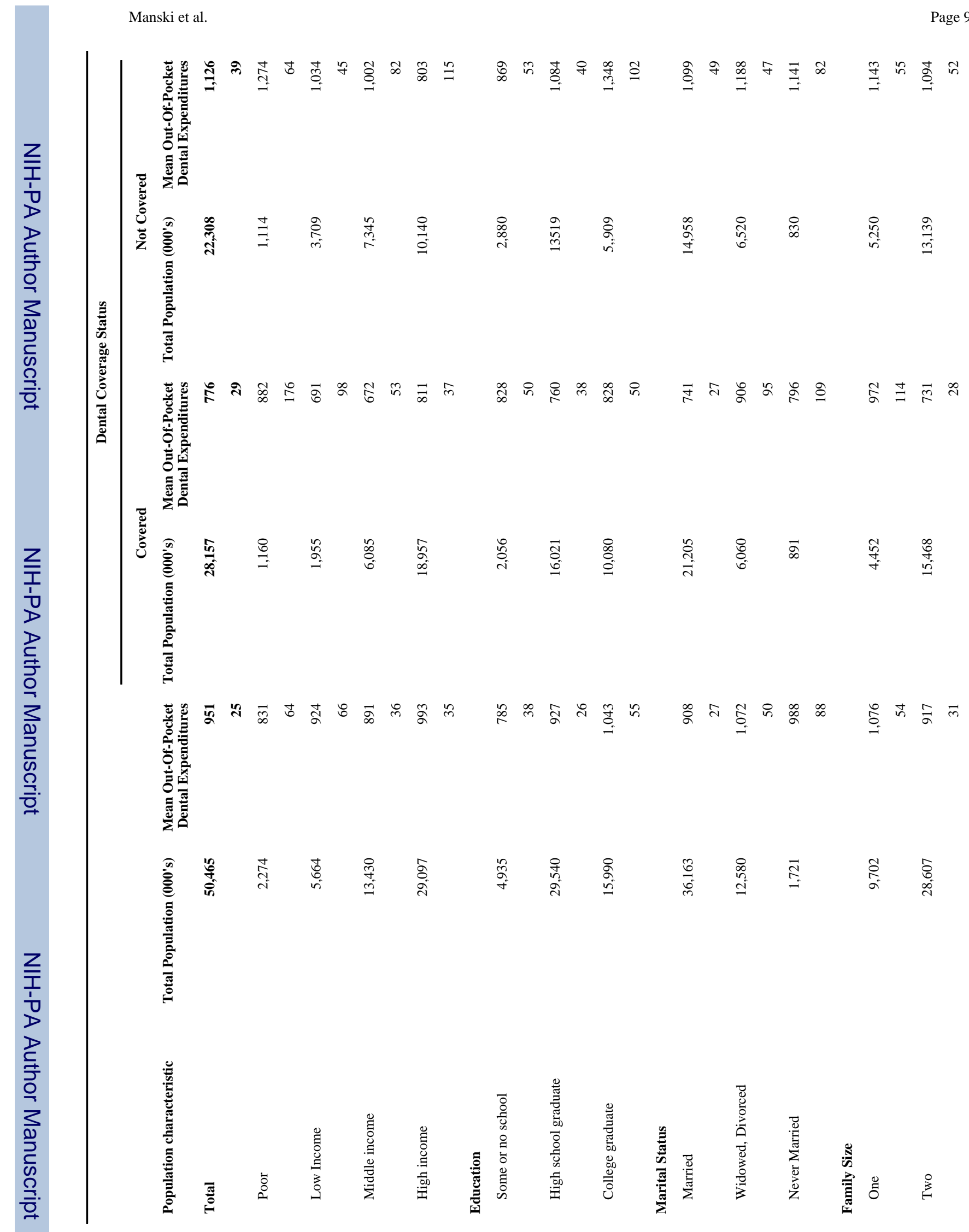

J Public Health Dent. Author manuscript; available in PMC 2010 October 19. 


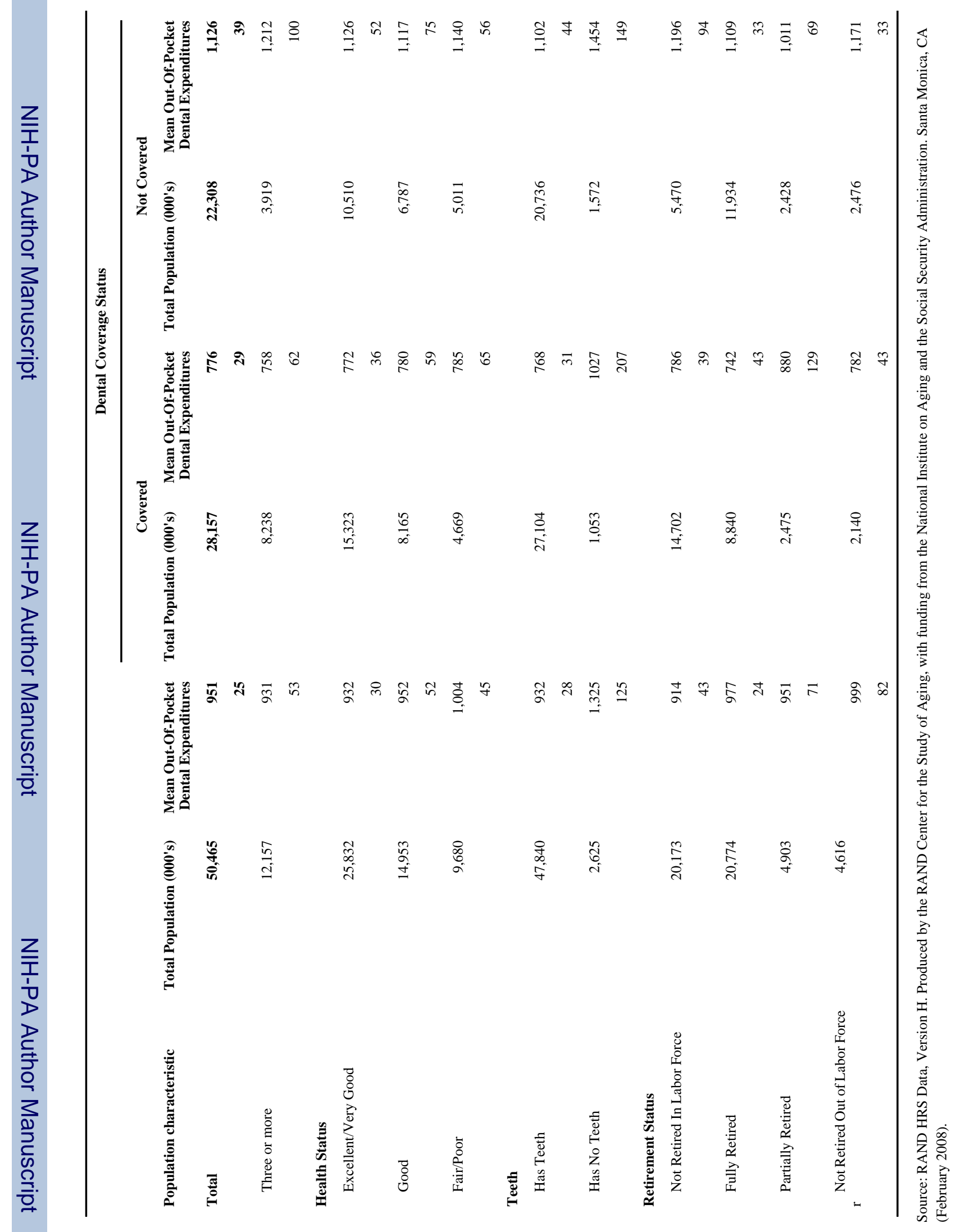




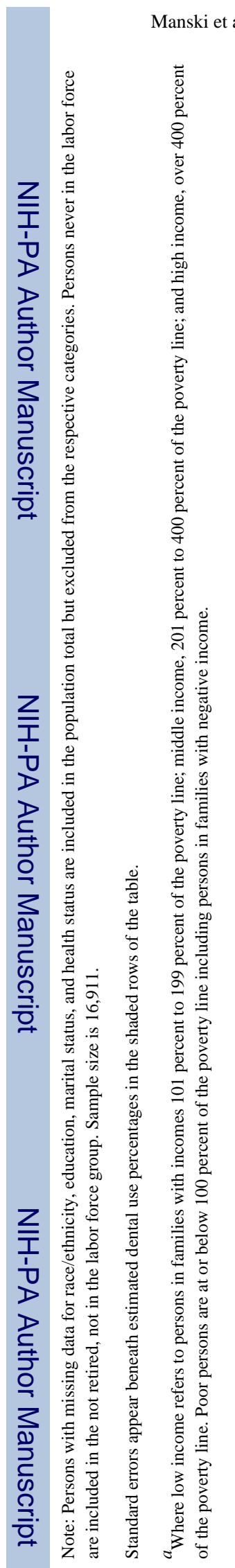

J Public Health Dent. Author manuscript; available in PMC 2010 October 19. 


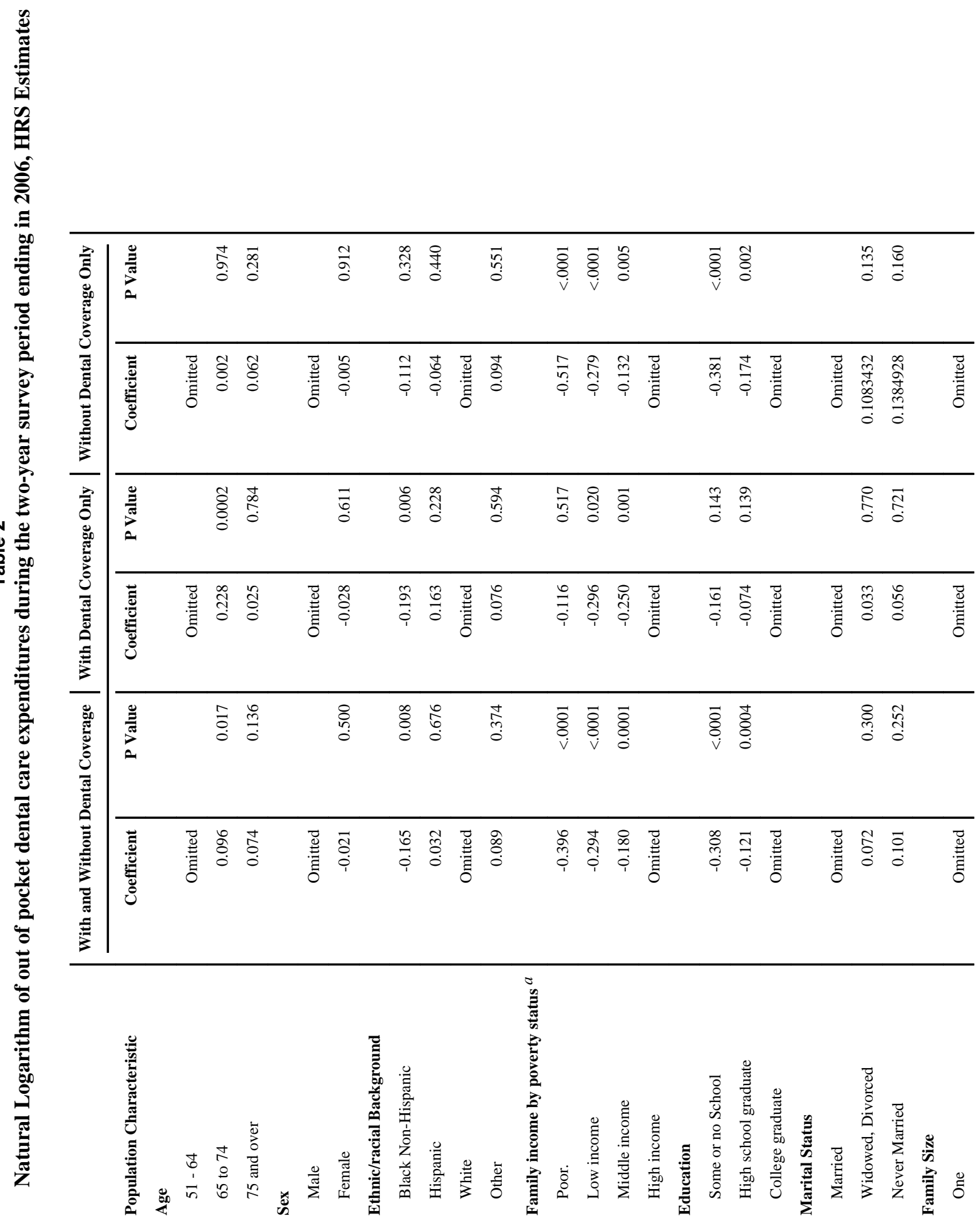




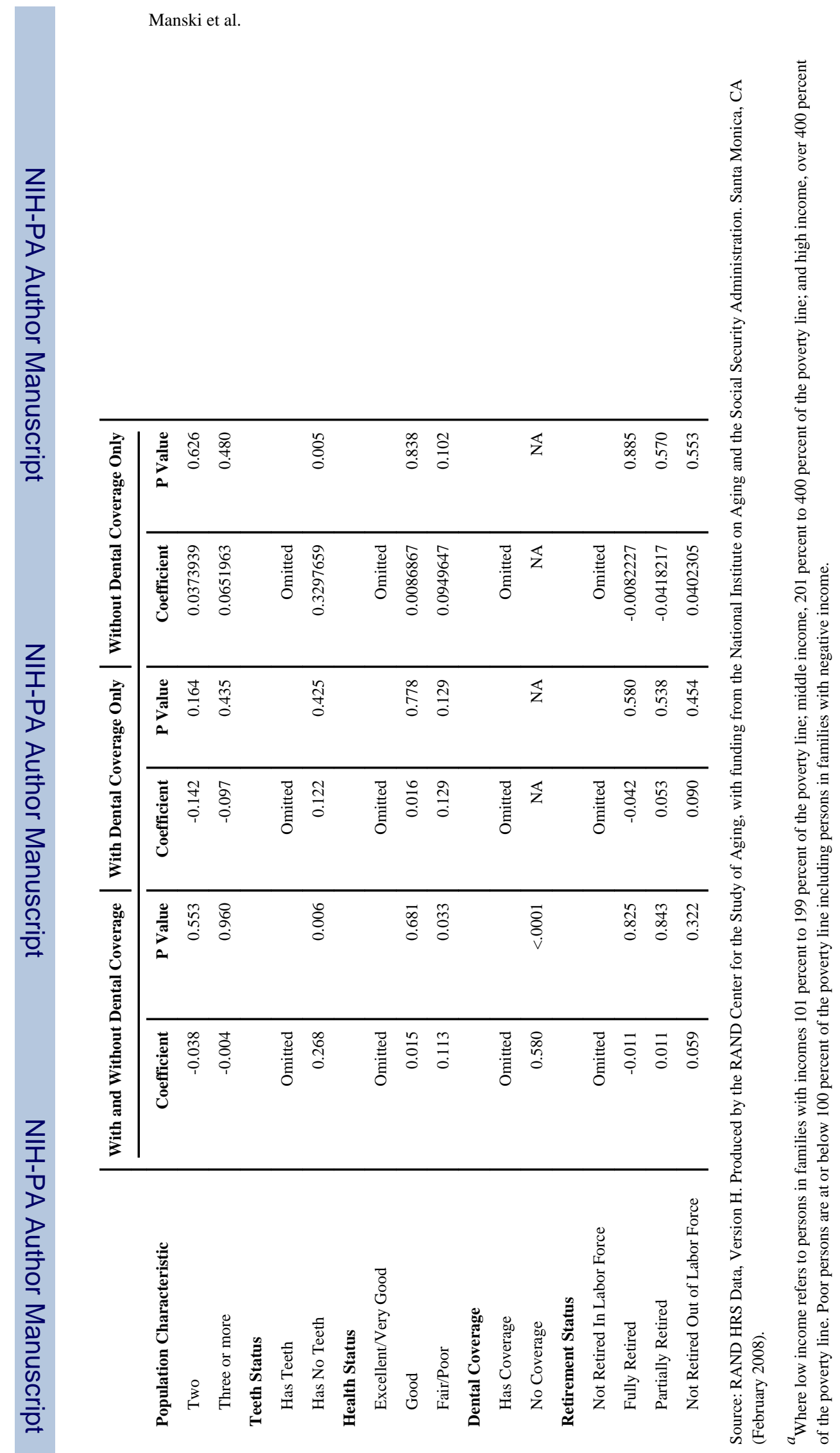

J Public Health Dent. Author manuscript; available in PMC 2010 October 19. 